\title{
Estimating long-range dependence in time series: An evaluation of estimators implemented in $\mathbf{R}$
}

\author{
Esther Stroe-Kunold, Tetiana Stadnytska, Joachim Werner, and Simone Braun \\ University of Heidelberg, Heidelberg, Germany
}

\begin{abstract}
Recent studies have shown that many physiological and behavioral processes can be characterized by long-range correlations. The Hurst exponent $H$ of fractal analysis and the fractional-differencing parameter $d$ of the ARFIMA methodology are useful for capturing serial correlations. In this study, we report on different estimators of $H$ and $d$ implemented in R, a popular and freely available software package. By means of Monte Carlo simulations, we analyzed the performance of (1) the Geweke-Porter-Hudak estimator, (2) the approximate maximum likelihood algorithm, (3) the smoothed periodogram approach, (4) the Whittle estimator, (5) rescaled range analysis, (6) a modified periodogram, (7) Higuchi's method, and (8) detrended fluctuation analysis. The findings_-confined to $\operatorname{ARFIMA}(0, d, 0)$ models and fractional Gaussian noise-identify the best estimators for persistent and antipersistent series. Two examples combining these results with the step-by-step procedure proposed by Delignières et al. (2006) demonstrate how this evaluation can be used as a guideline in a typical research situation.
\end{abstract}

\section{Serial Correlations of Processes in Psychological and Behavioral Research}

Recent empirical studies have demonstrated that many psychological, physiological, and behavioral time series exhibit significant long-range dependence (Aks \& Sprott, 2003; Delignières et al., 2006; Eke, Hermán, Kocsis, \& Kozak, 2002; Madison, 2004). Persistent autocorrelation implies a long memory of the data-generating process or, in other words, statistical dependence between observations separated by a large number of time units (Beran, 1994). In contrast, if a time series has a short memory and is predictable from only its immediate past, autocorrelations decay quickly as the number of intervening observations increases. Using spectral analysis techniques, Gilden and his colleagues demonstrated in experiments including mental rotation, lexical decision, shape and color discrimination, and visual search that serial autocorrelations accounted for even more variability in their data than did most standard manipulations in cognitive psychology (Gilden, 1997, 2001; Gilden, Thornton, \& Mallon, 1995; Gilden \& Wilson, 1995a, 1995b). Wagenmakers, Farrell, and Ratcliff (2004) confirmed these findings, employing in their analyses the ARFIMA methodology. Van Orden, Holden, and Turvey (2003), Wagenmakers et al. (2004), and Ward and Richard (2001) found longrange dependencies in automatic cognitive performance with measures such as word naming or simple reaction times. Chen, Ding, and Kelso (1997, 2001), Delignières, Lemoine, and Torre (2004), and Ding, Chen, and Kelso (2002) observed long-range correlations in human rhythmic activities such as tapping or in other tasks requiring coordination or synchronization of motor and cognitive activities. Delignières, Fortes, and Ninot (2004) reported long-range dependencies in time series of self-esteem and physical self. Long-range dependencies are also found in data based on human gait (Hausdorff, Zemany, Peng, \& Goldberger, 1999), force production tasks (Pressing, 1999), brain activity (Linkenkaer-Hansen, 2002), and heart rate fluctuations or other biological phenomena (Hausdorff \& Peng, 1996). A recent study has revealed the presence of long-range dependence in relative-phase series of bimanual coordination (see Torre, Delignières, $\&$ Lemoine, 2007b). Furthermore, Gilden and Hancock (2007) have provided new perspectives on cognitive assessments of attention-deficit disorders.

"Pink" or $1 / f$ noise represents one of the most prominent long-memory processes. As an intermediate between white noise (a process without memory) and brown noise (a process with an infinite memory), $1 / f$ noise could be conceived of as an indication of both the stability and the adaptability of a system. The concept of self-organized criticality (SOC), introduced by Bak, Tang, and Wiesenfeld (1987), illustrates these characteristics. A system is said to be selforganized when its structure emerges without explicit influences from outside the system. Thus, nonrandomness in the system emerges from random initial conditions and random input. The term self-organized implies this internal dynamic. The term criticality is analogous to the critical point of equilibrium systems. Thus, SOC characterizes systems that naturally evolve to a critical state in which a minor event starts a chain reaction that can affect any number of elements in the system (Bak, 1996). Such systems are said to have a critical point as an attractor. Long-range autocorrelations (pink noise or fractal dynamics) consti-

J. Werner, joachim.werner@psychologie.uni-heidelberg.de 
tute the typical signature of complex systems in a critical self-organized state. According to West and Shlesinger (1990) or Marks-Tarlow (1999), 1/f noise behavior is adaptive to both endogenous and exogenous perturbations and can be viewed as the typical signature of young, healthy, and adaptive systems. Hausdorff et al.'s (1997) report of $1 / f$ noise in the gait of healthy adults and behavior close to white noise for the elderly or for patients with Huntington's disorder provides empirical support for this assumption.

\section{Modeling Long-Range Dependence}

The basic properties of processes with long memory are a hyperbolically decaying autocorrelation function (ACF), a spectral density increasing without limit as the frequency tends to zero, and the so-called Hurst phenomenon. The last characterization implies that the Hurst exponent $(H)$, the parameter representing the probability that an event in a time series is followed by a similar event, deviates from .5. For $H=.5$, the observations are independent.

There are two classes of fractal processes, which can be persistent or antipersistent: fractional Brownian motion (fBm) and fractional Gaussian noise (fGn). Mandelbrot and van Ness (1968) introduced fBm as a generalization of

Fractional Gaussian Noise ( $\mathrm{fGn}$ )

$$
\begin{gathered}
\text { Antipersistent } \\
\begin{array}{rr}
\beta= & -0.50 \\
H= & .25 \\
d= & -0.25
\end{array}
\end{gathered}
$$

A

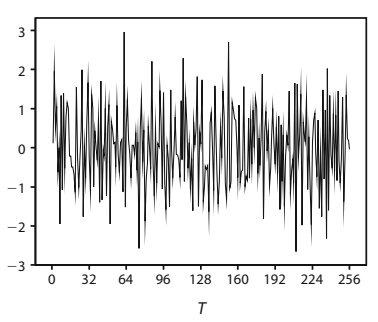

B

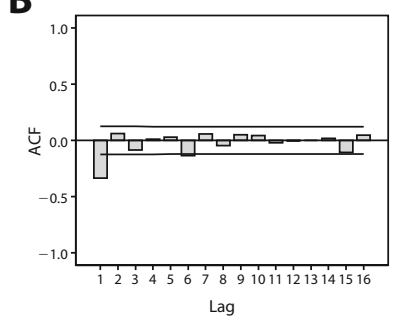

C

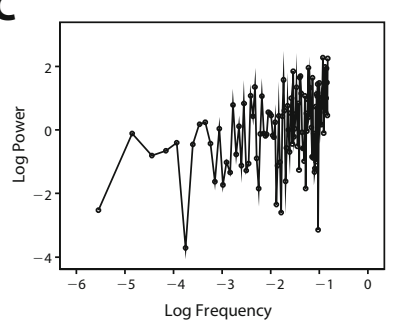

Persistent

$\beta=0.50$

$H=.75$

$d=0.25$
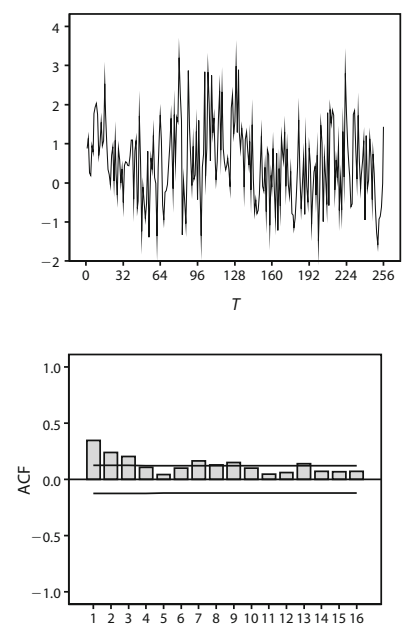

Lag

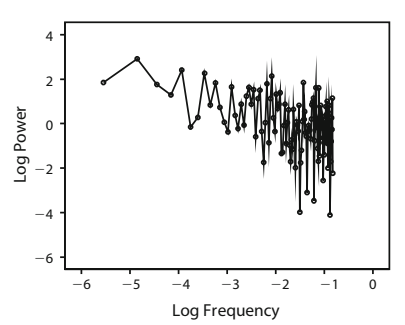

ordinary Brownian motion, a continuous-time stochastic process with independent increments. Brownian motion with $H=.5$ separates antipersistent and persistent $\mathrm{fBms}$. $H$ can be any real number in the range $[0, .5)$ for antipersistent and $(.5,1]$ for persistent series, where antipersistence implies negative correlations between the successive increments of a fBm series. fGn, a discrete-time analogue of fBm, was defined by Mandelbrot and Wallis (1969). Gaussian noise is a stationary process with constant mean and variance, whereas Brownian motion is nonstationary with stationary increments. Differencing $\mathrm{fBm}$ creates fGn, and summing fGn produces $\mathrm{fBm}$; the related processes are characterized by the same Hurst exponent.

There are three major ways for capturing long-range dependence: (1) by means of $H$ within the scope of fractal analysis; (2) through the power exponent $\beta$ of the power spectrum function $1 / f^{\beta}$ in the spectral analysis; and (3) through the fractional-differencing parameter $d$ within the ARFIMA framework. Figure 1 illustrates these concepts.

fBm and fGn are also called fractal noises. Fractal processes are self-similar long-memory processes. The concept of self-similarity, introduced into statistics by Mandelbrot and his coworkers (e.g., Mandelbrot \& van

\section{Fractional Brownian Motion (fBm)}

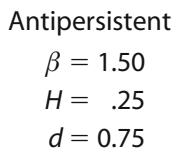
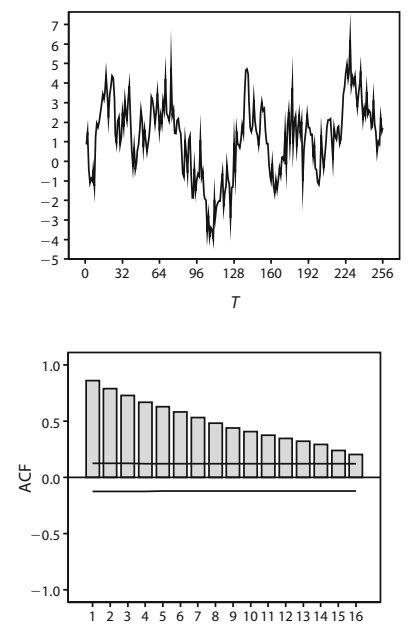

Lag

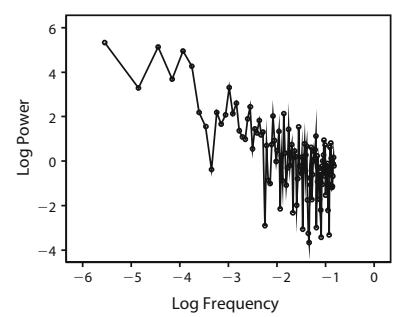

Persistent

$\beta=2.50$

$H=.75$

$d=1.25$
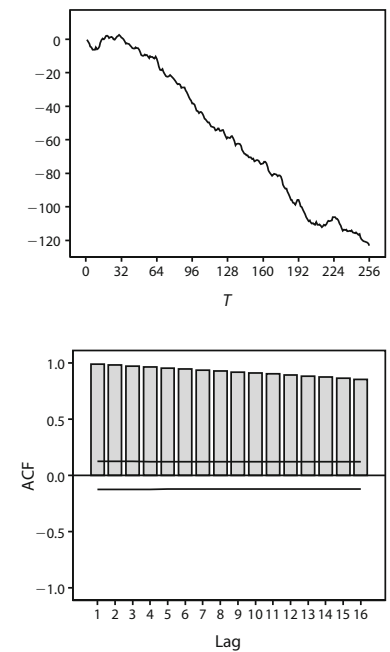

Lag

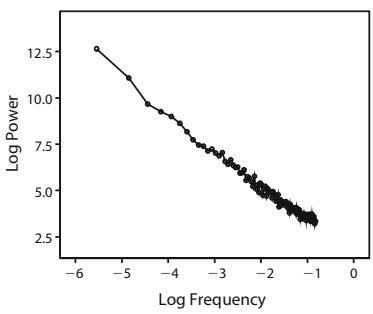

Figure 1. (A) The original series $(T=256)$, (B) autocorrelation functions (ACFs), and (C) spectral density plots. 
Ness, 1968; Mandelbrot \& Wallis, 1969), implies that a process appears roughly the same or possesses similar statistical features at different scales (see Beran, 1994, pp. 45ff, for details). Processes with fractal correlations have self-similar power spectra and autocorrelations. For long-range serial dependence, the power-spectral density of a process with serial dependence is proportional to the reciprocal of the frequency:

$$
S(f) \propto 1 / f^{\beta} .
$$

The power spectrum determines how much power $(S$; i.e., variance or amplitude) is accounted for by each frequency $(f)$ in the series. The parameter $\beta$ is called the power exponent: $\beta=0$ for ordinary Gaussian noise, $\beta=2$ for Brownian motion, and $\beta=1$ for pink or $1 / f$ noise. The power exponent of an fGn process can be any real value in the range $[-1,1]$, with $-1<\beta<0$ for antipersistent and $0<\beta<1$ for persistent series. For fBm, $1<\beta<2$ means an antipersistent and $2<\beta<3$ means a persistent series. In the spectral analysis, $\beta$ is estimated by calculating the negative slope of the line relating $\log S(f)$ and $\log f$; this method is called the power spectral density (PSD) analysis or the periodogram method. The following equations show how $\beta$ can be converted to $H$ (see, e.g., Eke et al., 2000):

$$
\begin{aligned}
& H=\frac{\beta+1}{2} \text { for } \mathrm{fGn} \\
& H=\frac{\beta-1}{2} \text { for } \mathrm{fBm} .
\end{aligned}
$$

Granger and Joyeux (1980) and Hosking $(1981,1984)$ demonstrated that self-similar hyperbolically decaying autocorrelations of fractional series can be parsimoniously modeled by means of the differencing parameter $d$ of the Box-Jenkins autoregressive integrated moving average (ARIMA) methodology by allowing it to take on continuous values. Thus, within the autoregressive fractionally integrated moving average [ARFIMA $(p, d, q)$ ] framework, $d$ is the parameter for modeling long-range dependence. The parameters $p$ and $q$ capture short-range dependencies of the process. The process is both stationary and invertible when $-0.5<d<0.5$. Values of $d$ in the interval $[-0.5,0]$ suggest antipersistence. Processes with $d>0$ are long-term persistent. Therefore, finite long memory can be modeled by $0<d<0.5$. For $0.5 \leq d \leq 1$, the process is nonstationary with noninvertible ARMA representations. Gaussian white noise, the process without memory, is the ARFIMA $(0,0,0)$ process. The ARFIMA
$(0,1,0)$ model represents random walk, the discrete-time analogue of ordinary Brownian motion. For fGn and increments of $\mathrm{fBm}$, the following relationships hold:

$$
\begin{aligned}
& \beta=2 d \\
& H=\frac{2 d+1}{2} .
\end{aligned}
$$

Table 1 summarizes the relationships between $\beta, H$, and $d$.

\section{Estimating Long-Range Dependence: \\ Review of Empirical Findings}

As described above, $\beta, d$, and $H$ are parameters showing the presence of serial dependence by means of the periodogram approach (i.e., spectral analysis or PSD regression), ARFIMA methodology, and fractal analysis. Bloomfield (2000) gave a detailed description of spectral analysis techniques, including methods for estimating $\beta$. Eke et al. (2000) and Delignières et al. (2006) demonstrated that, unlike many estimators of $H$ or $d$, the negative slope of the PSD regression provides a good estimate for both fBm and fGn series. Taqqu and Teverovsky (1998) showed the robustness of the periodogram method for series containing long-range as well as short-range dependence and for data with infinite variance innovations. The performance of PSD analysis was not extremely accurate for "ideal" series [fGn or ARFIMA $(0, d, 0)$ ], but it suffered from the smallest losses in efficiency as deviations from the "ideal" were introduced [e.g., innovations with an infinite variance, or $\operatorname{ARFIMA}(1, d, 1)]$. Thornton and Gilden (2005) developed, on the basis of PSD, a spectral classifier procedure that allowed distinguishing series with fractal correlations from those with short memory. In general, spectral techniques are susceptible to a range of statistical artifacts (see Press, Teukolsky, Vetterling, \& Flannery, 1992, for details). Nevertheless, they give a clear picture of fractal scaling in the low-frequency region of the spectral plot (Holden, 2005).

Wagenmakers, Farrell, and Ratcliff $(2004,2005)$ proposed an elaborate strategy for estimating $d$ in ARFIMA modeling. Farrell, Wagenmakers, and Ratcliff (2006) then conducted simulation experiments comparing the spectral classifier method with the ARFIMA approach. Both procedures proved to be equally effective in discriminating between long- and short-memory series. Torre, Delignières, and Lemoine (2007a) evaluated the ARFIMA method of Wagenmakers et al. $(2004,2005)$ and found this method appropriate to adequately detect long-range dependence in simulated fractal series.

Table 1

Relationships Between Parameters Capturing Long-Range Dependence

\begin{tabular}{lccc}
\hline & $\beta$ & $H$ & $d$ \\
\hline Random walk (ordinary Brownian motion) & 2 & .5 & 1 \\
White noise (ordinary Gaussian noise) & 0 & .5 & 0 \\
fGn & {$[-1,1]$} & {$[0,1]$} & {$[-0.5,0.5]$} \\
& {$[-1,0)$ antipersistent } & {$[0, .5)$ antipersistent } & {$[-0.5,0)$ antipersistent } \\
fBm & $(0,1]$ persistent & $(.5,1]$ persistent & $(0,0.5]$ persistent \\
& {$[1,3]$} & {$[0,1]$} & {$[0.5,1.5]$} \\
& {$[1,2)$ antipersistent } & {$[0, .5)$ antipersistent } & {$[0.5,1)$ antipersistent } \\
& $(2,3]$ persistent & $(.5,1]$ persistent & $(1,1.5]$ persistent \\
\hline
\end{tabular}


Different estimators for $\beta, d$, and $H$ have been evaluated in the time series literature in terms of spectral analysis, the ARFIMA methodology, and fractal analysis.

Eke et al. (2000) and Delignières et al. (2006) recommended lowPSD ${ }_{\mathrm{we}}$ (an improved variant of PSD analysis), the signal summation conversion method (SSC), or detrended fluctuation analysis (DFA) for distinguishing between $\mathrm{fGn}$ and $\mathrm{fBm}$. In addition, DFA has been evaluated as reliable and robust (Eke et al., 2002), does not require the arbitrary setting of parameters, and is not susceptible to most statistical artifacts, but it can falsely classify certain types of signals as fractal (see Rangarajan \& Ding, 2000, for details).

Stadnytska and Werner (2006) demonstrated that the conditional sum of squares (CSS) procedure, a computationally convenient technique available in current versions of SAS for Windows, and the exact maximum likelihood (EML) approach of Sowell (1992) provide very accurate estimates of $d$ for sample sizes of $1,000-1,600$ observations.

Taqqu and Teverovsky (1998) and Taqqu, Teverovsky, and Willinger (1995) described and evaluated various methods for estimating $d$ and $H$ for models with different innovation structures. The Whittle estimator of $d$ showed the best performance in the ARFIMA $(p, d, q)$ cases. For models with $d=0$, however, this method suffered from infrequent but very large outliers. Estimation methods such as rescaled range analysis $(\mathrm{R} / \mathrm{S})$, the variance method, or Whittle have tended to perform less well when there is additional short-range dependence structure in a series. The use of infinite- rather than finite-variance innovations, however, did not cause a great decline in their performance. The R/S approach, in particular, can be biased by slowly decaying trends in the time series (Beran, 1994).

Caccia, Percival, Cannon, Raymond, and Bassingthwaighte (1997), Cannon, Percival, Caccia, Raymond, and Bassingthwaighte (1997), Eke et al. (2000), Eke et al. (2002), and Delignières et al. (2006) systematically evaluated different classical fractal analysis methods for estimating $H$, including $\mathrm{R} / \mathrm{S}$, the scaled windowed variance method (SWV), and dispersional analysis (Disp). These studies revealed that most of these methods performed well within a given class (fGn or $\mathrm{fBm}$ ) but led to inconsistent results with the other class. For instance, SWV gave accurate estimates of $H$ for $\mathrm{fBm}$ and failed in the fGn case. In contrast, the Disp was appropriate only for fGn series. ${ }^{1}$ Therefore, the accurate estimation of $H$ requires the identification of the class of a series before the application of fractal analysis.

\section{Estimators Implemented in $\mathbf{R}$}

The goal of this study was to evaluate the performance of the estimators of $d, H$, and $\alpha$ implemented in $\mathrm{R}$ (R Development Core Team, 2008), a popular and freely available software package frequently used in the applied social and behavioral sciences. As compared with other software tools, R has many further advantages: It provides a variety of statistical and graphical techniques and is highly extensible, allowing users to add additional functionality by defining new functions. Various packages are under con- stant development for $\mathrm{R}$, covering a wide range of modern statistics.

This study is meant to support applied researchers who are not keen on designing complex programming or analyzing the algorithms behind it, and who therefore use only the default options. For this reason, the selection of the estimators we focus on here is restricted to the ones implemented in R.

Within the R framework, ARFIMA analysis can be performed by means of the fracdiff package, whereas the estimators of fractal analysis are implemented in the fractal package. The following $d$ estimators are available in fracdiff: (1) the Geweke-Porter-Hudak (1983) estimator, (2) the approximate maximum likelihood estimator (MLE) of Haslett and Raftery (1989), and (3) the smoothed periodogram approach of Reisen (1994). In the fractal package, (4) the Whittle estimator, (5) the R/S method, (6) the modified periodogram method, (7) Higuchi's (1988) estimator for $H$, and (8) the DFA approach are implemented.

fdGPH (fracdiff): Geweke-Porter-Hudak estimator. The semiparametric fdGPH algorithm estimates the long-memory parameter $d$ by means of the method proposed by Geweke and Porter-Hudak (1983). This well-known and widely used approach is based on the simple linear regression of the log periodogram on a deterministic regressor. The fdGPH is the ordinary least squares estimator of the slope parameter in this regression, formed using only the lowest-frequency ordinates of the log periodogram.

fdML (fracdiff): Approximate MLE. The ML estimator of $d$ implemented in $\mathrm{R}$ is an approximate method developed by Haslett and Raftery (1989; see also Brent, 1973). In contrast to other techniques implemented in fracdiff, this method also provides short-range dependency estimates of $p$ and $q$. The range of $d$ is confined to $[0,0.5]$ - that is, only persistent stationary series are considered.

fdSperio (fracdiff): Smoothed periodogram approach. The semiparametric fdSperio method, proposed by Reisen (1994; see also Reisen, Abraham, \& Toscano, 2000), estimates $d$ by means of the regression equation employing the smoothed periodogram function for modeling the spectral density. In contrast to the fdGPH and the fdML optimization algorithms, which constrain $d$ not to be greater than 0.5 , the fdSperio method is not restricted in this way. Therefore, it can be applied directly to nonstationary data.

fdWhittle (fractal): Whittle estimator. The fdWhittle algorithm estimates $d$ using the periodogram method. As described previously (see Equation 1), the spectral density of a series with long-range correlations should be proportional to the reciprocal of the frequency $\left(f^{-\beta}=f^{-2 d}=f^{1-2 H}\right)$. The relation $\beta=2 d$ holds for both fGn and fBm processes (see, e.g., Taqqu \& Teverovsky, 1998). Therefore, the Whittle estimator is appropriate for series with finite as well as infinite variance. The Whittle method estimates $d$, optimizing the function containing the quotient of the periodogram and the spectral density function at different frequencies. For further details, consult Beran (1994), Fox and Taqqu (1986), or Taqqu and Teverovsky (1998). 
RoverS (fractal): R/S method. The RoverS approach estimates $H$ by means of the $\mathrm{R} / \mathrm{S}$ method, introduced by Hurst, Black, and Simaika (1965) and described in detail by Mandelbrot and Wallis (1969) and Mandelbrot (1975). The series is divided into nonoverlapping intervals of length $n$. Within each interval, the partial sum of a time series is computed. The R/S statistic is obtained as a quotient relating the difference between the maximum and the minimum of the partial sum and the standard deviation of the original series. For fGn, the following relationship holds:

$$
E(\mathrm{R} / \mathrm{S}) \propto n^{H} \text { as } n \rightarrow \infty .
$$

Therefore, the estimate of $H$ is obtained as the slope of the averaged $\mathrm{R} / \mathrm{S}$ as a function of $n$ and is appropriate only for fGn signals.

hurstSpec (fractal): Modified periodogram method. The hurstSpec method estimates $H$ via spectral regression using the relationship between $\beta$ and the Hurst coefficient, as given in Equation 2A. Therefore, this estimator is confined to fGn signals. This approach is a modification of the periodogram method that compensates for the fact that most of the frequencies on a log-log plot fall on the far right, and thus exert a strong influence on the least-square line fitted to the periodogram. In this method, the frequency axis is divided into logarithmically equally spaced boxes, and the periodogram values corresponding to the frequencies inside each box are averaged. According to Taqqu et al. (1995), several of the values at very low frequencies are left untouched, since there are so few of them to begin with.

hurstBlock (fractal): Higuchi's method. The hurstBlock algorithm estimates $H$ in the time domain - that is, using the sample values of the time series (not the spectrum). Within the hurstBlock routine, several estimation methods are available. Using absolute values of the aggregated series (method="aggabs") is the default. Systematic preliminary simulations have shown, however, that the Higuchi approach (method="higuchi"; Higuchi, 1988) clearly outperforms the other methods. This is probably due to the fact that the Higuchi method evaluates the cumulative sum of the data to convert the series from a noise to a motion. Therefore, this is the approach considered in our study. The basic principle underlying this method is calculating the length of a path and finding its fractal dimension $D$. The original series is divided into blocks of size $n$, and the partial sum within each block is calculated (e.g., generating $\mathrm{fBm}$ from $\mathrm{fGn}$ ). $H$ is obtained by means of $D$, since for the normalized length of the curve $[L(n)]$, the following relationship holds:

$$
E[L(n)] \propto n^{-D},
$$

where $D=2-H$.

DFA (fractal): Detrended fluctuation analysis. The DFA method, originally proposed by Peng et al. (1993), estimates the scaling exponent $\alpha$, which can be converted into $H$ using the following equations:

$$
\begin{array}{ll}
H=\alpha & \text { for fGn } \\
H=\alpha-1 & \text { for fBm. }
\end{array}
$$

The estimate of $\alpha$ is obtained by means of the following steps: After the integration of the time series, the integrated series is divided into nonoverlapping intervals of length $n$. A least-square line (i.e., the trend of the interval) is fit to the data in each of these intervals. The series is locally detrended by subtracting the theoretical values provided by the regression. For all possible interval lengths, the characteristic size of fluctuation $(F)$ for the integrated and detrended series is calculated. $F$ increases with the interval length according to the following power law:

$$
F \propto n^{\alpha} .
$$

$\alpha$ is the slope of a double logarithmic plot of $F$ as a function of $n$, ranging within $[0,0.5)$ for antipersistent and $(0.5,1]$ for persistent fGn. For fBm, $1<\alpha<1.5$ implies antipersistence, and $1.5<\alpha<2$ implies a persistent series. For Brownian noise, $\alpha$ is 1.5 .

The DFA approach allows for distinguishing between fGn and fBm signals. $\alpha$ exponents ranging from 0 to 1 indicate $\mathrm{fGn}$, whereas $\mathrm{fBm}$ corresponds to exponents ranging from 1 to 2 (see Delignières et al., 2006).

Using R, two specifications have to be made (apart from the default command) in order to obtain interpretable results. The command sum.order $=1$ ensures that each series is integrated first. Furthermore, systematic preliminary simulations have shown that only bridge detrendingalso used in the PSD approach - provides good results for fBm series. ${ }^{2}$ Thus, in the present article, we used an improved version of DFA analysis, applying the R command DFA(x, detrend="bridge", sum.order $=1$ ).

\section{MONTE CARLO STUDY Evaluation of Estimators Implemented in R}

The following Monte Carlo study aimed at comparing and evaluating the different estimators of the memory parameter in ARFIMA $(0, d, 0)$ models implemented in $\mathrm{R}$.

\section{Method}

Generating the sample via Monte Carlo simulations. There are two options within the $\mathrm{R}$ framework to simulate fractionally integrated time series. The fractal package includes the option 1 Simulate, simulating a stochastic fractal time series via the Davies-Harte (1987) technique. This method for generating exact fractional Gaussian noise is widely used and is based on the fast Fourier transform algorithm. For details, consult, for instance, Beran (1994), Caccia et al. (1997), and Delignières et al. (2006). In addition, the fracdiff package contains the option fracdiff.sim to simulate fractional ARIMA times series via the same algorithm. The first method simulates the series for a given $H$, and the second one for a given $d$. The R code by Peter F. Craigmile, available at www.stat .osu.edu/ pfc/software/, provides deeper insight into the Davies-Harte algorithm (see also Craigmile, 2003). For the following Monte Carlo study, we simulated ARFIMA $(0, d, 0)$ models employing both options. Because no discrepancies in the results were observed, we describe only the results obtained from the fracdiff.sim routine. 
Study design. The performance of the estimators was assessed on six ARFIMA $(0, d, 0)$ models for variations of $d$ ranging from $-0.45,-0.3,-0.1$ (antipersistent fGn) to $0.1,0.3,0.45$ (persistent fGn), respectively, for variations of $H$ ranging from $.05, .2, .4$ (antipersistent fGn) to $.6, .8, .95$ (persistent fGn). Different variations of $T(256,512,1,024$, and 2,048) were considered. All simulations were conducted for 1,000 replications. We computed the mean values over 1,000 replications for estimates of $d$ and $H$, as well as the corresponding standard deviations and mean squared errors $\left(M S_{\mathrm{e}}\right)$. As a quality criterion, we computed the percentage of correct estimations within the intervals $\left[d_{\text {true }}-0.05, d_{\text {true }}+\right.$ $0.05]$ and $\left[H_{\text {true }}-.05, H_{\text {true }}+.05\right]$, respectively. For detailed analysis, we also calculated the percentages of overand underestimations. One might argue that the intervals we chose are quite narrow, leading to a strict evaluation of the estimators. Nevertheless, it became obvious that some of the estimators still performed quite well under these conditions. Furthermore, an over- or underestimation of .05 can lead to remarkable differences in interpretation of the results. All computations were performed with $\mathrm{R}$ version 2.6.2.

\section{Results}

Figure 2A illustrates the percentages of over-, under-, and correct estimates of $d$ and $H$ for the different types of estimators for antipersistent fGn. Figure 2B gives the same information for persistent fGn.

Figure 3 summarizes, for both persistent and antipersistent series, the $M S_{\mathrm{e}}$ values of $\hat{d}$ and $\hat{H}$ obtained from the different methods and sample sizes.

For antipersistent and persistent series, the fdGPH method did not perform accurately: The amount of correct estimates
$T$

256

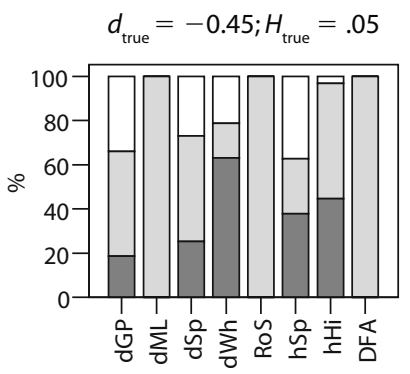

512

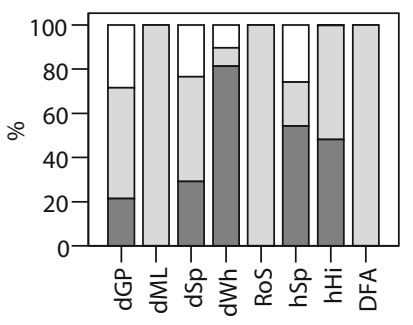

1,024

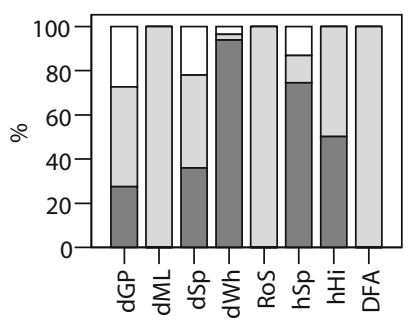

2,048

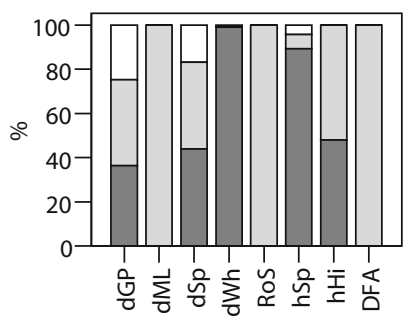

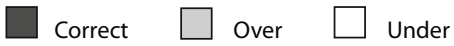
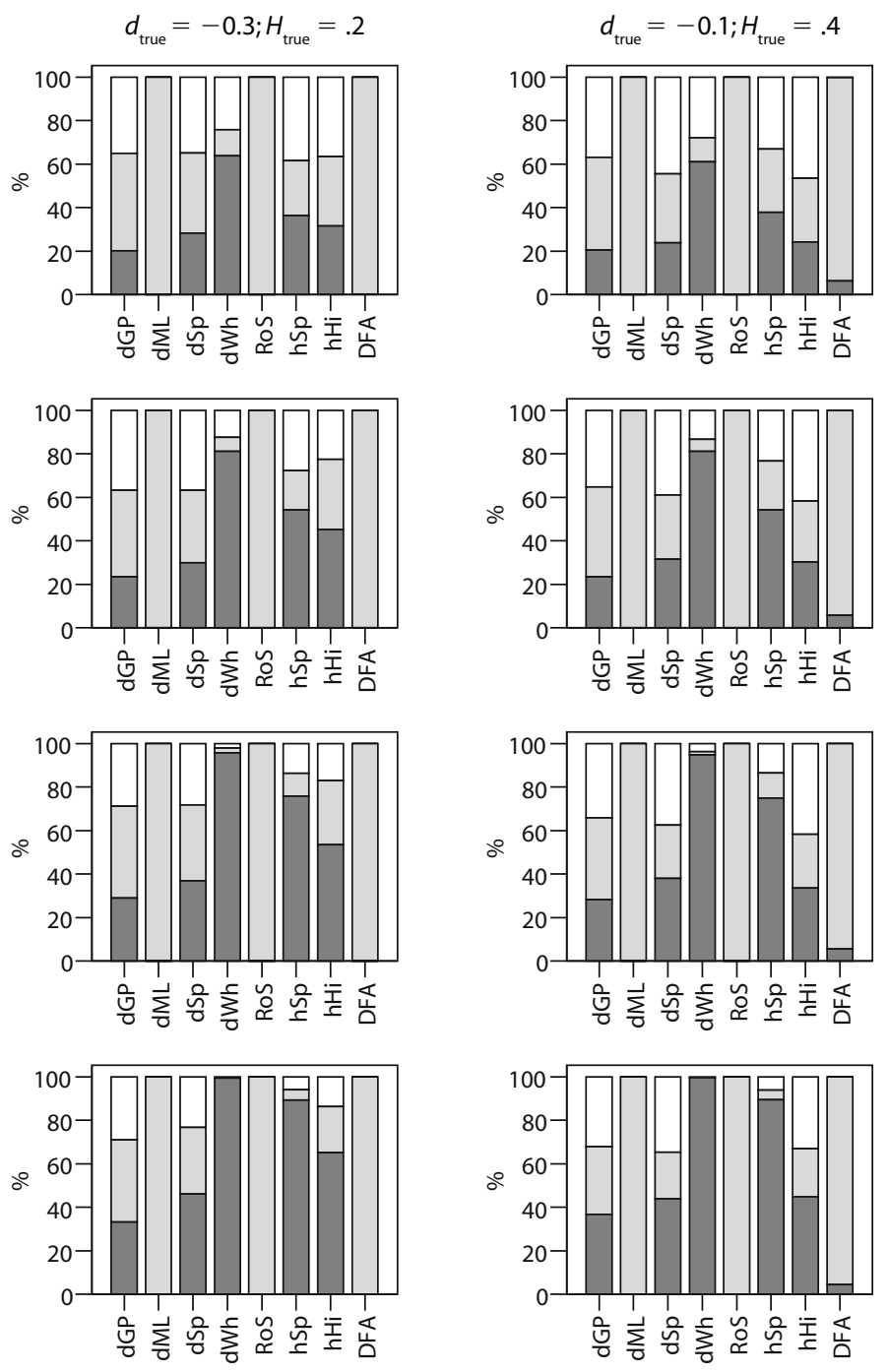

Figure 2A. Antipersistent fGn series. Percentages of over- (i.e., $x_{\text {est }}>x_{\text {true }}+0.05$ ), under- (i.e., $x_{\text {est }}<x_{\text {true }}-0.05$ ), and correct (i.e., $\left.x_{\text {est }} \in\left[x_{\text {true }}-0.05, x_{\text {true }}+0.05\right]\right)$ estimates of $d$ and $H$ for the different types of estimators and variations of $T$ (1,000 replications). Abbreviations: dGP, fdGPH; dML, fdML; dSp, fdSperio; dWh, fdWhittle; RoS, RoverS; hSp, hurstSpec; hH, hurstBlock (method = "higuchi"). 
$T$

256

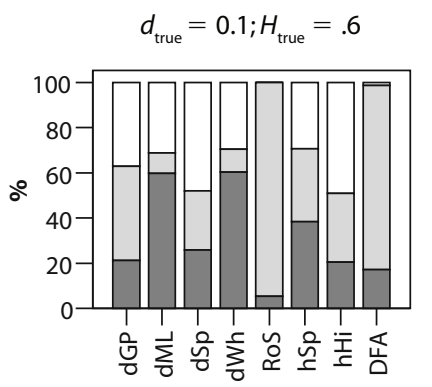

512

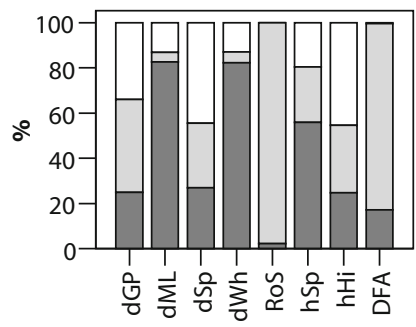

1,024

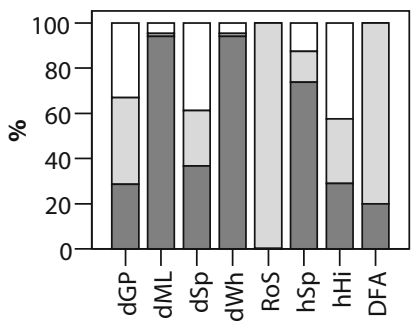

2,048

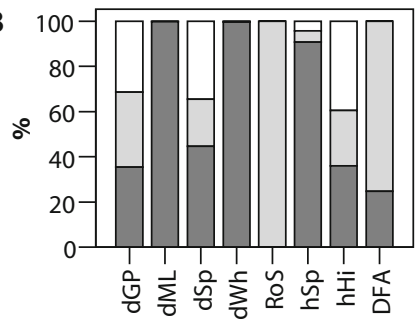

Correct $\square$ Over $\square$ Under

$d_{\text {true }}=0.3 ; H_{\text {true }}=.8$
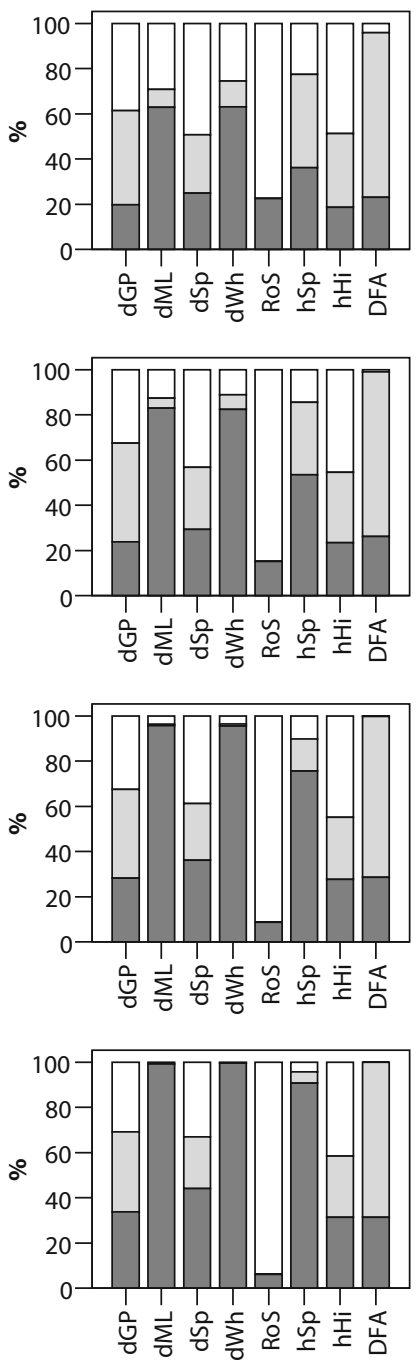

$d_{\text {true }}=0.45 ; H_{\text {true }}=.95$
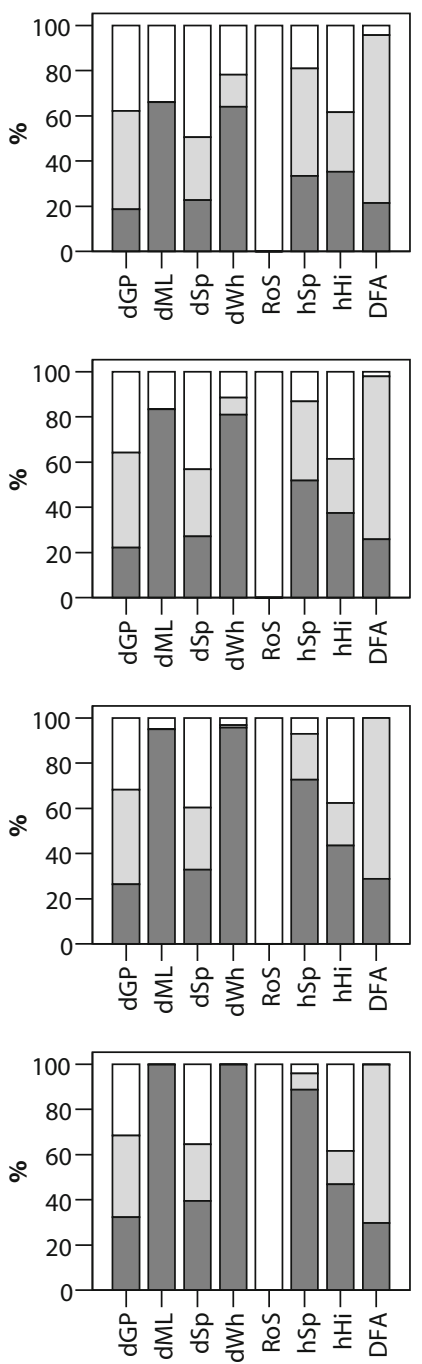

Figure 2B. Persistent fGn series. Percentages of over-, under-, and correct estimates of $\boldsymbol{d}$ and $\boldsymbol{H}$ for the different types of estimators. See Figure 2A for further explanations.

was less than $40 \%$ for all variations of $d$ and $T$. For small sample sizes, there was a tendency toward overestimation. The $M S_{\mathrm{e}}$ values increased for decreasing $T$ but remained quite high in comparison with the other estimators evaluated. Note that similar results were obtained for fdSperio.

As described previously, the range of fdML is restricted to $[0, .5]$. Therefore, it failed completely in the antipersistent case. For persistent fGn, this estimator improved its performance with increasing $T$, independent of the $d$ parameterization. In small samples, there was a slight tendency toward underestimation. Still, fdML clearly outperformed other methods, with around $60 \%$ correct estimates for $T=256$. The percentages of correct identifications were over $80 \%$ for $T=512$, almost $100 \%$ for $T=1,024$, and approximately $100 \%$ for $T=2,048$. Extremely low $M S_{\mathrm{e}}$ values confirmed the convincing performance of this estimator for persistent fGn.
The fdWhittle estimator clearly delivered the highest rate of correct identifications among all of the estimators for $d$. With a small sample size $(T=256)$, the amount of correct decisions was around $60 \%$ for all variations of $d$, with a slight tendency toward underestimation. The other percentages of correct identifications were around $80 \%$ for $T=512$, close to $100 \%$ for $T=1,024$, and approximately $100 \%$ for $T=2,028$. The $M S_{\mathrm{e}}$ values were extremely low.

The performance of the RoverS estimator was extremely poor. In the antipersistent case, it overestimated the true parameters. For persistent series, different nonsystematic biases were observed, generating inaccurate results. These results were not consistent with the findings of Eke et al. (2000) and Delignières et al. (2006), who evidenced a slight overestimation bias for antipersistent fGn, but nevertheless obtained values of $H<.5$. This discrepancy can be explained by the fact that the latter studies used an improved 


\section{Antipersistent fGn}
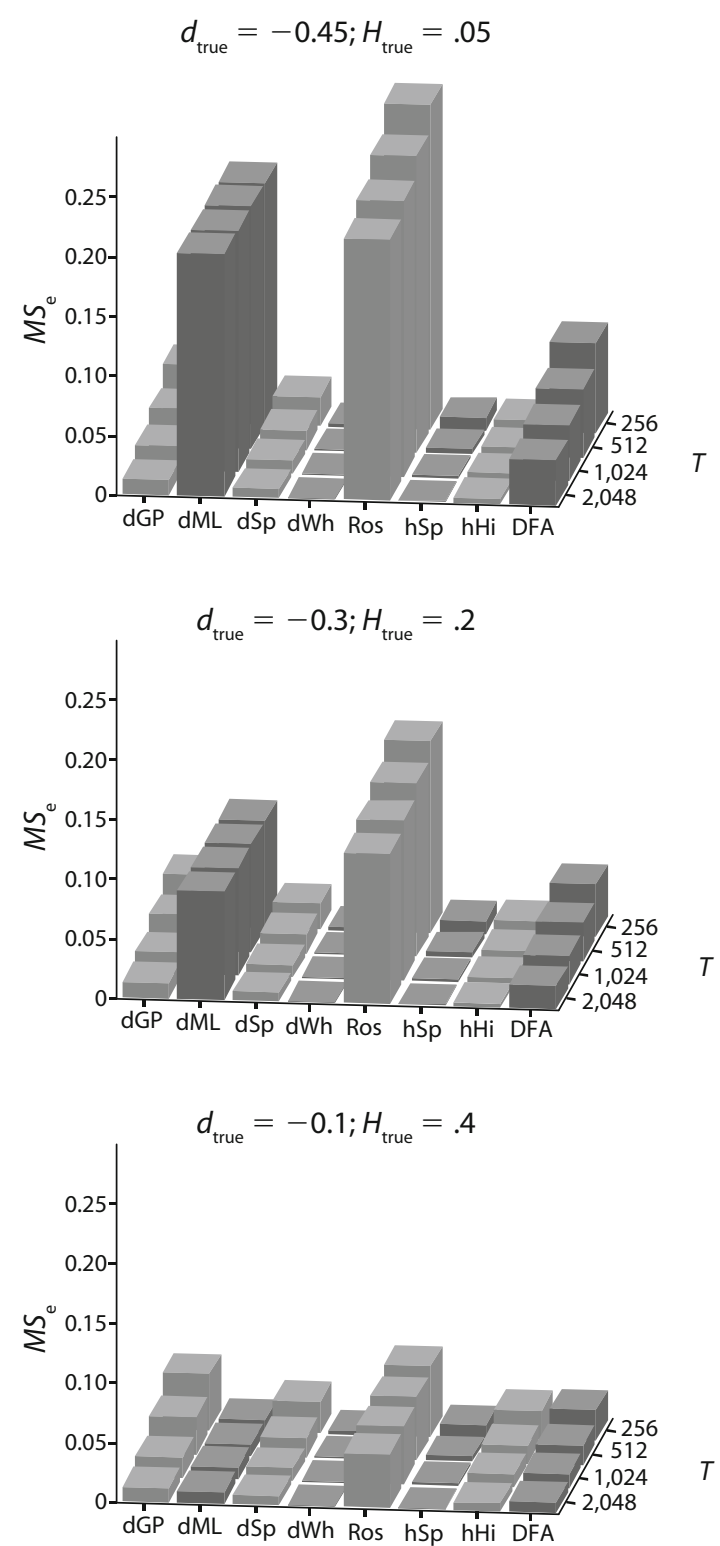

\section{Persistent $\mathrm{fGn}$}

$$
d_{\text {true }}=0.1 ; H_{\text {true }}=.6
$$
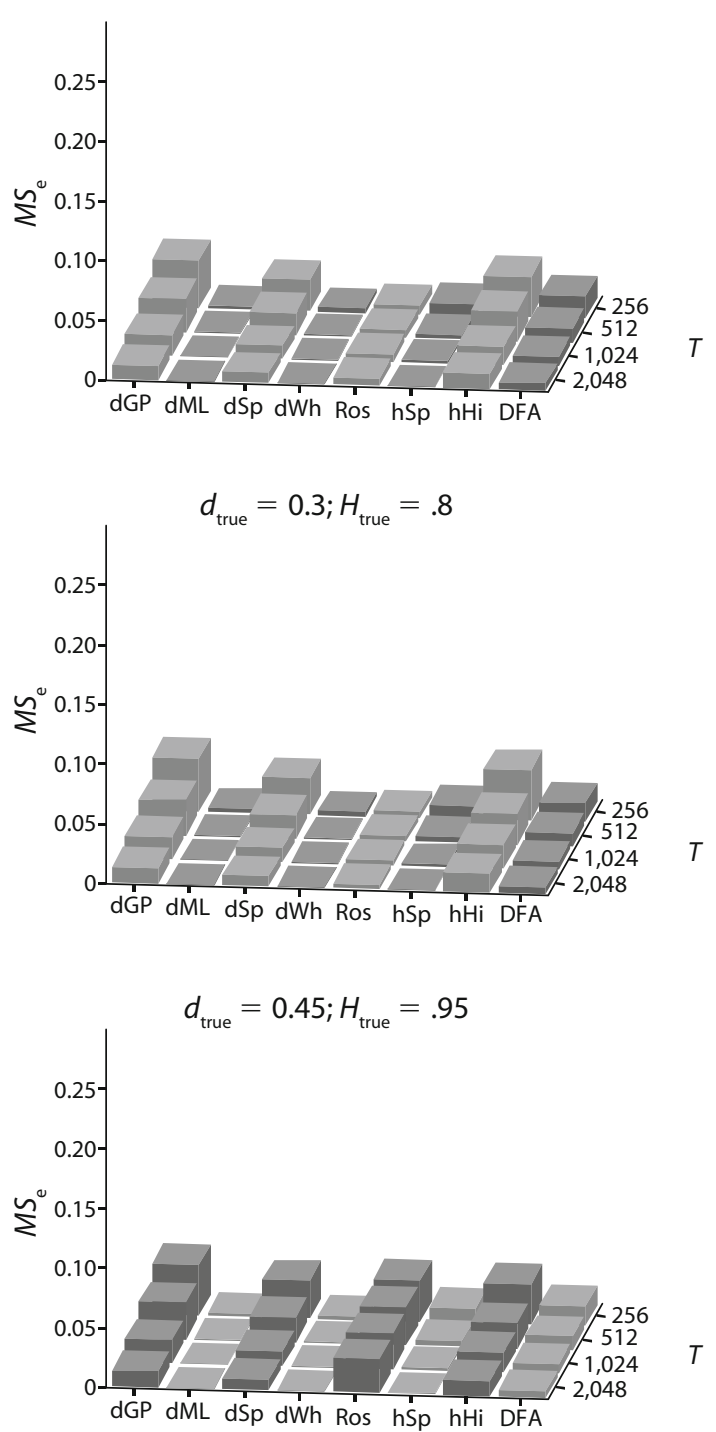

Figure 3. $M S_{\mathrm{e}}$ for the estimates of $d$ and $H$ (over 1,000 replications) for the different types of estimators and variations of $T$ (third dimension). See Figure $2 \mathrm{~A}$ for abbreviations.

version of the rescaled range analysis, the so-called $R / S$ detrended method. As mentioned above, the present article is designed for applied researchers who use the default options implemented in R. The RoverS estimator of $\mathrm{R}$ allows only a few manipulations - for example, of the number of blocks. The option of detrending is not included.

The hurstSpec method was the best estimator of $H$. For antipersistent series, the percentage of correct identifications improved as the sample size increased: slightly under $40 \%$ for $T=256$, almost $60 \%$ for $T=512$, about $80 \%$ for $T=1,024$, and around $90 \%$ for $T=2,048$. For persistent series, comparable results were obtained. The hurstSpec estimates provided the smallest $M S_{\mathrm{e}}$ values.
The performance of the Higuchi estimator depended on the $d$ parameterizations. For $H_{\text {true }}=.05$, there was a tendency toward overestimation, with almost $50 \%$ correct decisions, independent of the sample size. For $H_{\text {true }}=.2$, performance improved with increasing $T$ (from around $30 \%$ correct identifications for $T=256$ to over $60 \%$ for $T=2,048)$. For $H$ values with $.4, .6$, and .8 , the amount of correct estimates increased slightly with larger sample sizes (around $20 \%$ for $T=256$ but slightly over $40 \%$ for $T=2,048$ ) - in this case, however, with a tendency toward underestimation. For $H_{\text {true }}=.95$, the percentages of correct decisions were almost $40 \%$ with the smaller samples $(T=256$ or 512$)$ and over $40 \%$ with the larger samples 
( $T=1,024$ or 2,048). Apart from RoverS, the Higuchi method achieved the highest $M S_{\mathrm{e}}$ values among the estimators of $H$.

For antipersistent series, the results for the DFA approach were not at all convincing, with a global overestimation of $\alpha$. For persistent fGn, performance was slightly better, with around $30 \%$ correct estimates for $T=2,048$. Still, the percentage of overestimation was extremely high. The estimates of $\alpha$ lay in the range between 0 and 1 . Thus, our simulation study confirmed the finding that the DFA method allows for distinguishing between fGn and fBm (see the details above).

To sum up, fdWhittle was the most accurate estimator of $d$, independent of parameterizations and sample sizes. For persistent series, the performance of fdML was comparable to that of fdWhittle. For estimating $H$, hurstSpec was the best method. Finally, the DFA approach can be used a priori to distinguish between $\mathrm{fGn}$ and $\mathrm{fBm}$.

\section{EXAMPLES}

The following examples aim at combining these findings with the step-by-step procedure proposed by Delignières et al. (2006) and demonstrating how this evaluation can be used as a guideline in a typical research situation. We first present the analysis of a series gained by means of a temporal estimation experiment, which was used for the first time by Gilden (e.g., 1997, 2001) to study $1 / f$ noise. This is followed by the reanalysis of a glacial varve series described in Shumway and Stoffer (2006).

For interested researchers, the $\mathrm{R}$ code used to analyze the series can be found in the Appendix, but it is also available at www.psychologie.uni-heidelberg.de/ae/meth/ stroe-kunold.html. Here, they also find the temporal estimation data set. The varve series can be obtained from www.stat.pitt.edu/stoffer/tsa2/.

As a first step of the following analyses, we distinguished between fGn and fBm signals by means of DFA analysis as well as by low $\mathrm{PSD}_{\mathrm{we}}$, an improved version of the PSD method, as recommended by Delignières et al. (2006). The next steps were ARFIMA and fractal analyses using the estimators evaluated above.

\section{Temporal Estimation Task}

The first series, of length $T=512$, was obtained via an experiment conducted by means of the Presentation software (see www.neurobs.com). The particular setup of our experiment was based on the temporal estimation task used by Wagenmakers et al. (2004). The stimulus was an hourglass presented at the center of a black computer screen until a response was registered. A practice phase of 24 stimuli preceded the experimental phase, which included 512 stimuli. The task was to estimate 1,000msec time intervals. The participant was instructed to press the space key with the first finger of the right hand to indicate when he or she thought that $1,000 \mathrm{msec}$ had elapsed since stimulus onset. Afterward, the number of milliseconds that had passed between stimulus onset and pressing the key was presented as a number on the black screen (true feedback). No specific instructions were given with respect to counting (see also Gilden, 2001). As in Wagenmakers et al.'s (2004) experiment, participants were instructed to keep their finger on the response key throughout the experiment, to minimize noise due to motor processes.

Figure 4 shows the plot of the original series, the autocorrelation function (ACF), and the double logarithmic plot of power against frequency obtained from the ${ }^{\text {low }} \mathrm{PSD}_{\text {we }}$ analysis.
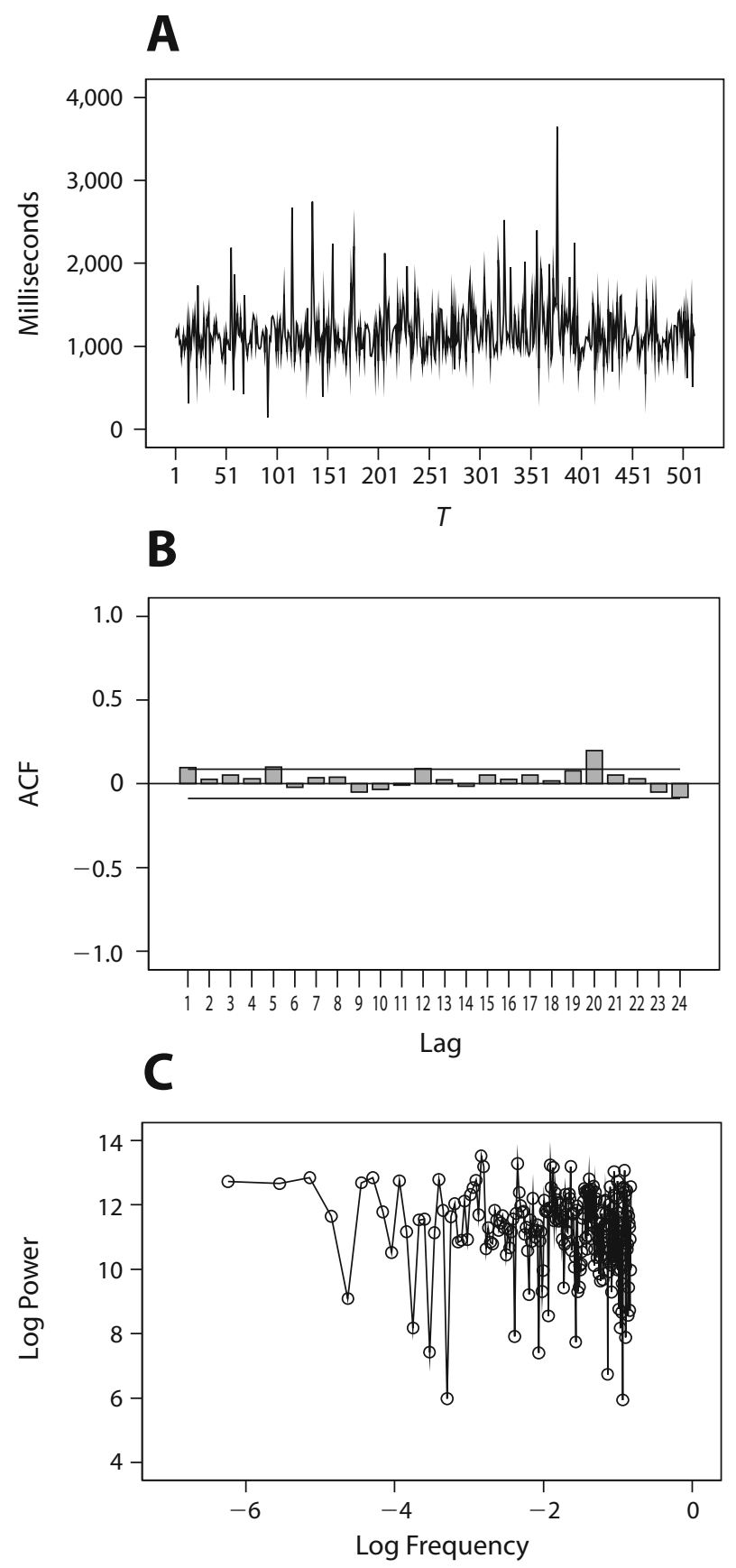

Figure 4. Empirical example of a temporal estimation task. The panels show the original series $(\mathrm{A})$, its autocorrelation function (ACF) (B), and the double logarithmic plot of power against frequency obtained from the low $P S D_{\text {we }}$ analysis (C). 
Both the plot and the ACF suggest the stationarity of the series. The DFA analysis indicates that the series is fGn $(\hat{\alpha}=0.68)$. Furthermore, the estimate of $\beta=.19$ obtained through the lowPSD $\mathrm{we}_{\text {we }}$ analysis confirms this impression; therefore, the series could be modeled as Gaussian. This analysis also provides the first estimates $d \approx 0.1$ and $H \approx .6$.

With regard to the ARFIMA analysis, we first consider the fdML estimator, since it performs best for persistent series. In the ARFIMA $(0, d, 0)$ case, $\hat{d}$ was 0.09 . This method also provides short-range dependency estimates of $p$ and $q$. Thus, in the ARFIMA $(1, d, 1)$ case, we obtained $\hat{d}=0.17, A R=0.6$, and $M A=0.7$. The latter result implies that the series is not ideally Gaussian, but contains long-range as well as short-range dependencies. In addition, the fact that the series is relatively short could influence the accuracy of estimates of $d$ and $H$. The results of our study suggest that the fdGPH method $(\hat{d}=0.39$, $s=0.17$ ) probably overestimates the true parameter, since this tendency was observed in series with $T=512$ (see Figure 2B). Note that in contrast to fdGPH, the fdSperio approach showed no tendency toward overestimation and provided an estimate less than $0.3(\hat{d}=0.24, s=0.04)$. It is possible, though, that the accuracy of fdSperio was affected by short-range dependencies. In sum, from the ARFIMA analysis, we conclude that the true value of $d$ can be expected to fall between 0.09 and 0.17 , because the fdML estimator provided the most reliable results in our evaluation study. This conclusion is also supported by the fractal analysis, since the most reliable estimators gave the following results: $\hat{d}=0.09$ (fdWhittle) and $\hat{H}=.59$ (hurstSpec). We cannot yet conclude from this that the series is necessarily a slightly persistent fGn series. Validating this claim would require that a persistent and an antipersistent model be tested against each other. Concerning model selection in general, the ARFIMA approach and the spectral classifier method are the most prominent procedures proposed in the literature. Wagenmakers et al. (2004) suggested determining the maximum likelihood of a time series under the ARFIMA (given a hypothesis of long-range dependence) and ARMA (given an alternative hypothesis of short-range dependence) models, and then selecting the appropriate representation using Akaike's information criterion. Thornton and Gilden (2005), on the other hand, proposed the spectral classifier procedure, in which the likelihood of a time series is estimated by comparing its power spectrum with the spectra of the competing memory models. For a detailed comparison of both methods, consult Farrell et al. (2006).

The fact that the data do not yield a robust signal can be explained by the inclusion of trial feedback. It seems that trial feedback works as a perturbation of trial performance that contributes unsystematic variation and whitens the $1 / f$ signal.

\section{Reanalysis of a Glacial Varve Series (Shumway \& Stoffer, 2006)}

In addition, we reanalyzed freely available data for a glacial varve series given in Shumway and Stoffer (2006, pp. 274ff). Sedimentary deposits (so-called varves) are used as proxies for paleoclimatic parameters (e.g., temperature). The series under investigation here consists of the thickness of the yearly varves collected from one location in Massachusetts for 634 years (i.e., $T=634$ ). The original series was log-transformed to remove nonstationarity in the variance as a function of time.

Figure 5 shows plots of the original and the logtransformed series, the ACF of the transformed series, and the double logarithmic plot of power against frequency obtained via the low $\mathrm{PSD}_{\mathrm{we}}$ analysis.

In contrast to the example described above, the plot of the transformed series, as well as its ACF, does not allow us to exclude nonstationarity. There could be some trends in the series, and the ACF decays very slowly, implying stronger long-range dependence. With its $\hat{\alpha}$ of 1.01 , the DFA analysis confirms that the series is at the limits of nonstationarity. The lowPSD ${ }_{\text {we }}$ analysis, with its estimate of $\beta=.72$, allows the series to be modeled as Gaussian. This implies first estimates of $d \approx 0.36$ and $H \approx .86$. Within the scope of ARFIMA methodology, we consider fdML to be the most reliable estimator, providing $\hat{d}=0.39$. Note that the fdGPH estimate $(\hat{d}=0.49, s=0.1)$ is also clearly higher in this case than the fdML estimate (as in the previous example). fdWhittle and hurstSpec, the best methods within the framework of fractal analysis, support the fdML estimation, with $\hat{d}=0.4$ and $\hat{H}=.97$, respectively.

As can be seen above, the $\mathrm{R}$ output only provides the standard error deviations of the fdGPH and the fdSperio estimates. The Monte Carlo simulation showed, however, that standard errors are quite independent of the different parameterizations. By averaging the standard errors over the different variations of the parameters, the simulation results can provide standard errors for the different estimators depending on $T$. These are listed in Table 2 .

According to the simulation study, the estimates are approximately normally distributed over all replications. Therefore, it seems legitimate to use Table 2 to apply an inferential test to the empirical results.

As can be seen from Table 3, almost all estimates for both data sets are highly significant- that is, $d$ is significantly different from 0 , and $H$ and $\alpha$ are significantly different from 0.5 (null hypothesis of short-range dependence). Therefore, the results support the assumption of long-range dependence in the temporal estimation series as well as the glacial varve series. For a rigorous statistical proof, the method of Wagenmakers et al. (2004) or Thornton and Gilden (2005) should be applied.

\section{CONCLUSIONS}

The fact that this study aims at supporting applied researchers, thus restricting its evaluation to estimators implemented in R, might limit the generality of the results found here, because their naive application could have misleading consequences for researchers' insights into psychological phenomena.

First, the shortcomings of the methods themselves have to be considered. Most of the tools presented here are adapted from linear analysis. Thus, their compatibility with fractal phenomena needs to be questioned. Recently, 


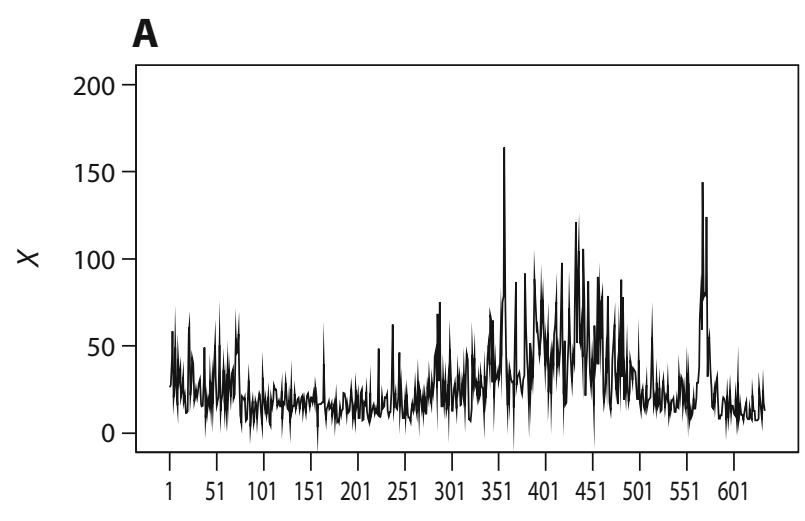

\section{B}

$T$

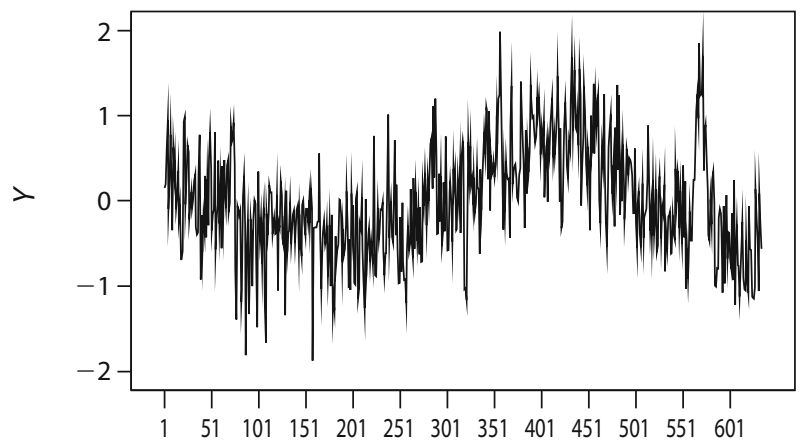

C
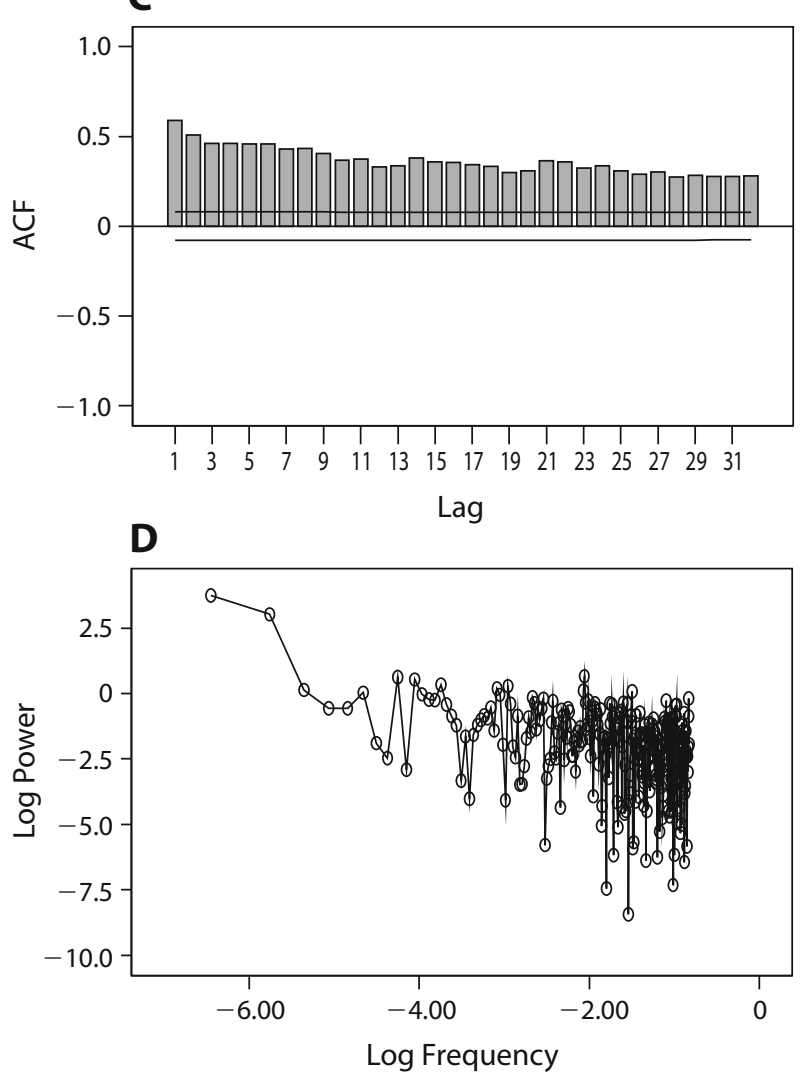

Figure 5. Empirical example of a glacial varve series. The panels show the original series (A), the log-transformed series (B), the autocorrelation function (ACF) of the log-transformed series (C), and the double logarithmic plot of power against frequency obtained from the ${ }^{\text {low }} \mathrm{PSD}_{\mathrm{we}}$ analysis (D). 
Table 2

Standard Deviations of Estimates of $\boldsymbol{d}, \boldsymbol{H}$, and $\boldsymbol{\alpha}$, Averaged Over All Parameter Variations Used in the Monte Carlo Experiment (i.e., for fGn Series, Over 1,000 Replications)

\begin{tabular}{|c|c|c|c|c|c|c|c|c|}
\hline & dGP & $\mathrm{dML}$ & $\mathrm{dSp}$ & $\mathrm{dWh}$ & RoS & $\mathrm{hSp}$ & hHi & DFA \\
\hline$T=256$ & 0.21 & 0.05 & 0.16 & 0.05 & 0.02 & 0.10 & 0.15 & 0.07 \\
\hline$T=512$ & 0.17 & 0.03 & 0.13 & 0.04 & 0.01 & 0.06 & 0.12 & 0.05 \\
\hline$T=1,024$ & 0.14 & 0.02 & 0.11 & 0.03 & 0.01 & 0.04 & 0.10 & 0.03 \\
\hline$T=2,048$ & 0.11 & 0.02 & 0.09 & 0.02 & 0.01 & 0.03 & 0.08 & 0.03 \\
\hline
\end{tabular}

Table 3

Estimated Values for $\boldsymbol{d}, \boldsymbol{H}$, and $\boldsymbol{\alpha}$ for a Time Series Gained via a Temporal Estimation Task and for the Glacial Varve Series

\begin{tabular}{|c|c|c|c|c|c|c|c|c|}
\hline & dGP & dML & $\mathrm{dSp}$ & $\mathrm{dWh}$ & RoS & $\mathrm{hSp}$ & hHi & DFA \\
\hline Temporal estimation & $0.39^{*}$ & $0.09^{* *}$ & $0.24^{* * *}$ & $0.09^{*}$ & $0.67^{* * *}$ & 0.59 & $1.01^{* * *}$ & $0.68^{* * *}$ \\
\hline Glacial varve & $0.49^{* * *}$ & $0.39^{* * *}$ & $0.49^{* * *}$ & $0.40^{* * *}$ & $0.72^{* * * *}$ & $0.97^{* * *}$ & $1.01^{* * *}$ & $1.01^{* * *}$ \\
\hline
\end{tabular}

nonlinear methods have increasingly been explored in the social and behavioral sciences (consult Riley \& Van Orden, 2005, for a detailed discussion and overview; for an example, see Zbilut \& Marwan, 2008). As illustrated in the empirical examples, an elaborate strategy to compensate for these limitations would be to combine different complementary tools estimating fractal dimension, such as PSD plots and DFA. Holden (2005) recommended combining these with dispersional analysis (Bassingthwaighte, Liebovitch, \& West, 1994), because the strengths of each of these analyses can compensate for the weaknesses of the others (see also Delignières, Torre, \& Lemoine, 2005).

Second, the researcher's perspective should go far beyond the issue of the mere precision of a measurement. Regardless of how much effort is invested in refining methods, estimators of long-range persistence in data should not be treated as providers of true values. The dynamic linkage between the component systems of a human being, as well as between human beings and their environments, has to be thoroughly taken into consideration (for a detailed discussion, consult Van Orden, Kello, \& Holden, in press). Future studies will show whether new approaches, such as fractional cointegration (e.g., Maddala \& Kim, 1998), can adequately capture dynamic process systems with long memory by modeling and analyzing them together (for an introduction to cointegration, consult StroeKunold \& Werner, 2008). Our statistical evaluation of estimators by means of Monte Carlo simulations represents a first step before interpreting the parameters on the level of contents (e.g., in the framework of a concrete empirical study). Searching for explanations of the phenomena and developing methodologies for accurate estimation of the long-range parameters are thus two necessary and complementary focal points in the actual discussion on long-range dependence.

\section{GENERAL DISCUSSION}

The scope of this study was limited to fGn and ARFIMA $(0, d, 0)$ models. Future research will be necessary to eval- uate the performance of these estimators for $\mathrm{fBm}$ series and for more complex ARFIMA $(p, d, q)$ structures. Another issue of interest will be a systematic comparison of different algorithms simulating long-memory processesfor example, the method based on the so-called Cholesky decomposition (see, e.g., Beran, 1994).

In the present study, we evaluated estimators in terms of their default settings in $\mathrm{R}$ because the majority of empirical researchers probably use those settings first. As a next step, additional tools provided in $\mathrm{R}$ for refining estimation of the parameters characterizing long-range dependence could be analyzed. Furthermore, estimators implemented in other software packages, such as OxMetrics or SAS, could be compared with those available in R. Moreover, extensive research is necessary to test the new approaches for modeling long-range dependence that have recently been developed. For instance, Beran and colleagues have proposed semiparametric fractional autoregressive models, which allow researchers to decide simultaneously whether an observed process contains a stationary short- or long-memory component, a difference stationary component, and/or a deterministic trend component (see Beran \& Feng, 2002; Beran \& Ocker, 1999).

\section{AUTHOR NOTE}

The research for this article was supported by a scholarship awarded the first author by the German National Academic Foundation. Part of this work was done while the first author was a visiting scholar with EricJan Wagenmakers at the University of Amsterdam. The authors thank him and his lab for helpful hints and comments. In addition, this article has profited from correspondence with Donald Percival (University of Washington, Seattle) as well as with Sofiane Ramdani (University of Montpellier). We especially thank Guy Van Orden (University of Cincinnati) for enriching comments on the manuscript. Correspondence related to this article may be sent to J. Werner, Department of Psychology, University of Heidelberg, Hauptstr. 47-51, 69117 Heidelberg, Germany (e-mail: joachim.werner@psychologie.uni-heidelberg.de).

\section{REFERENCES}

Aкs, D. J., \& Sprotт, J. C. (2003). The role of depth and $1 / f$ dynamics in perceiving reversible figures. Nonlinear Dynamics, Psychology, \& Life Sciences, 7, 161-180. 
BAK, P. (1996). How nature works: The science of self-organized criticality. New York: Copernicus.

BAK, P., TANG, C., \& Wiesenfeld, K. (1987). Self-organized criticality: An explanation of the $1 / f$ noise. Physical Review Letters, 59, 381384

Bassingthwaighte, J. B., Liebovitch, L. S., \& West, B. J. (1994). Fractal physiology. New York: Oxford University Press.

Beran, J. (1994). Statistics for long-memory processes. New York: Chapman \& Hall.

BerAn, J., \& FENG, Y. (2002). SEMIFAR models-A semiparametric approach to modelling trends, long-range dependence and nonstationarity. Computational Statistics \& Data Analysis, 40, 393-419.

BERAN, J., \& OCKER, D. (1999). SEMIFAR forecasts, with applications to foreign exchange rates. Journal of Statistical Planning \& Inference, 80, 137-153.

Bloomfield, P. (2000). Fourier analysis of time series: An introduction (2nd ed.). New York: Wiley.

BRENT, R. P. (1973). Algorithms for minimization without derivatives. Englewood Cliffs, NJ: Prentice Hall.

Caccia, D. C., Percival, D., Cannon, M. J., Raymond, G., \& Bassingthwaighte, J. B. (1997). Analyzing exact fractal time series: Evaluating dispersional analysis and rescaled range methods. Physica A, 246, 609-632.

Cannon, M. J., Percival, D. B., Caccia, D. C., Raymond, G. M., \& Bassingthwaighte, J. B. (1997). Evaluating scaled windowed variance methods for estimating the Hurst coefficient of time series. Physica A, 241, 606-626.

Chen, Y., Ding, M., \& Kelso, J. A. S. (1997). Long memory processes $\left(1 / f^{a}\right.$ type $)$ in human coordination. Physical Review Letters, 79, 4501-4504.

Chen, Y., Ding, M., \& Kelso, J. A. S. (2001). Origins of time errors in human sensorimotor coordination. Journal of Motor Behavior, 33, 3-8.

Craigmile, P. F. (2003). Simulating a class of stationary Gaussian processes using the Davies-Harte algorithm, with application to long memory processes. Journal of Time Series Analysis, 24, 505-511.

DAVIES, R. B., \& HARTE, D. S. (1987). Tests for Hurst effect. Biometrika, 74, 95-101.

Delignières, D., Fortes, M., \& Ninot, G. (2004). The fractal dynamics of self-esteem and physical self. Nonlinear Dynamics, Psychology, \& Life Sciences, 8, 479-510.

Delignières, D., Lemoine, L., \& Torre, K. (2004). Time intervals production in tapping and oscillatory motion. Human Movement Science, 23, 87-103.

Delignières, D., Ramdani, S., Lemoine, L., Torre, K., Fortes, M., \& NinOT, G. (2006). Fractal analyses for "short" time series: A reassessment of classical methods. Journal of Mathematical Psychology, 50, 525-544.

Delignières, D., Torre, K., \& Lemoine, L. (2005). Methodological issues in the application of monofractal analyses in psychological and behavioral research. Nonlinear Dynamics, Psychology, \& Life Sciences, 9, 435-461

Ding, M., Chen, Y., \& Kelso, J. A. S. (2002). Statistical analysis of timing errors. Brain \& Cognition, 48, 98-106.

Eke, A., Hermán, P., Bassingthwaighte, J. B., Raymond, G. M., Percival, D. B., Cannon, M., Et AL. (2000). Physiological time series: Distinguishing fractal noises from motions. Pflügers Archiv, 439, 403-415.

Eke, A., Hermán, P., Kocsis, L., \& KozaK, L. R. (2002). Fractal characterization of complexity in temporal physiological signals. Physiological Measurement, 23, R1-R38.

Farrell, S., Wagenmakers, E.-J., \& RatclifF, R. (2006). 1/f noise in human cognition: Is it ubiquitous, and what does it mean? Psychonomic Bulletin \& Review, 13, 737-41.

Fox, R., \& TAQQU, M. S. (1986). Large-sample properties of parameter estimates for strongly dependent stationary Gaussian time series. Annals of Statistics, 14, 517-532.

GeweKe, J., \& Porter-HudaK, S. (1983). The estimation and application of long memory time series models. Journal of Time Series Analysis, 4, 221-238.

GILDEN, D. L. (1997). Fluctuations in the time required for elementary decisions. Psychological Science, 8, 296-301.

GiLden, D. L. (2001). Cognitive emissions of $1 / f$ noise. Psychological Review, 108, 33-56.
GILDEN, D. L., \& HANCOCK, H. (2007). Response variability in attentiondeficit disorders. Psychological Science, 18, 796-802.

Gilden, D. L., Thornton, T., \& Mallon, M. W. (1995). $1 / f$ noise in human cognition. Science, 267, 1837-1839.

GiLDEN, D. L., \& Wilson, S. G. (1995a). On the nature of streaks in signal detection. Cognitive Psychology, 28, 17-64.

GILDEN, D. L., \& WiLSON, S. G. (1995b). Streaks in skilled performance. Psychonomic Bulletin \& Review, 2, 260-265.

Granger, C. W. J., \& Joyeux, R. (1980). An introduction to longmemory time series models and fractional differencing. Journal of Time Series Analysis, 1, 15-29.

Haslett, J., \& Raftery, A. E. (1989). Space-time modelling with longmemory dependence: Assessing Ireland's wind power resource. $A p$ plied Statistics, 38, 1-50.

Hausdorff, J. M., Mitchell, S. L., Firtion, R., Peng, C. K., CudkoWICZ, M. E., WeI, J. Y., \& GoldBERgER, A. L. (1997). Altered fractal dynamics of gait: Reduced stride-interval correlations with aging and Huntington's disease. Journal of Applied Physiology, 82, 262-269.

Hausdorff, J. M., \& Peng, C. (1996). Multiscaled randomness: A possible source of $1 / f$ noise in biology. Physical Review E, 54, 2154 2157.

Hausdorff, J. M., Zemany, L., Peng, C., \& Goldberger, A. L. (1999) Maturation of gait dynamics: Stride-to-stride variability and its temporal organization in children. Journal of Applied Physiology, 86, 1040-1047.

HiguchI, T. (1988). Approach to an irregular time series on the basis of the fractal theory. Physica D, 31, 277-283.

HoldEN, J. G. (2005). Gauging the fractal dimension of response times from cognitive tasks. In M. A. Riley \& G. C. Van Orden (Eds.), Tutorials in contemporary nonlinear methods for the behavioral sciences (pp. 268-318). [Online] Retrieved October 7, 2008, from www.nsf .gov/sbe/bcs/pac/nmbs/nmbs.jsp.

Hosking, J. R. M. (1981). Fractional differencing. Biometrika, 68, 165 176 .

Hosking, J. R. M. (1984). Modeling persistence in hydrological time series using fractional differencing. Water Resources Research, 20, 1898-1908.

HuRST, H. E., Black, R. P., \& Simaika, Y. M. (1965). Long-term storage, an experimental study. London: Constable.

LinKENKAER-HANSEn, K. (2002). Self-organized criticality and stochastic resonance in the human brain. Unpublished doctoral dissertation, Helsinki University of Technology, Helsinki, Finland.

Maddala, G. S., \& Kim, I.-M. (1998). Unit roots, cointegration, and structural change. Cambridge: Cambridge University Press.

MADISON, G. (2004). Fractal modeling of human isochronous serial interval production. Biological Cybernetics, 90, 105-112.

Mandelbrot, B. B. (1975). Limit theorems on the self-normalized range for weakly and strongly dependent processes. Zeitschrift für Wahrscheinlichkeitstheorie \& verwandte Gebiete, 31, 271-285.

Mandelbrot, B. B., \& van Ness, J. W. (1968). Fractional Brownian motion, fractional noises and applications. SIAM Review, 10, 422-437.

Mandelbrot, B. B., \& Wallis, J. R. (1969). Computer experiments with fractional Gaussian noises. Water Resources Research, 5, 228 267.

Marks-Tarlow, T. (1999). The self as a dynamical system. Nonlinear Dynamics, Psychology, \& Life Sciences, 3, 311-345.

Peng, C.-K., Mietus, J., Hausdorff, J. M., Havlin, S., Stanley, H. E., \& GoldBerger, A. L. (1993). Long-range anticorrelations and non-Gaussian behavior of the heartbeat. Physical Review Letters, 70 , 1343-1346.

Press, W. H., Teukolsky, S. A., Vetterling, W. T. \& Flannery, B. P. (1992). Numerical recipes in C: The art of scientific computing (2nd ed.). Cambridge: Cambridge University Press.

Pressing, J. (1999). Sources for $1 / f$ noise effects in human cognition and performance. In R. Heath, B. Hayes, A. Heathcote, \& C. Hooker (Eds.), Proceedings of the Fourth Conference of the Australian Cognitive Science Society. Newcastle, NSW: University of Newcastle, Department of Psychology.

Rangarajan, G., \& Ding, M. (2000). First passage time distribution for anomalous diffusion. Physics Letters A, 273, 322-330.

R Development Core Team (2008). R: A language and environment for statistical computing. Vienna: R Foundation for Statistical Computing. Available at www.R-project.org. 
REISEN, V. A. (1994). Estimation of the fractional difference parameter in the ARIMA $(p, d, q)$ model using the smoothed periodogram. Journal of Time Series Analysis, 15, 335-350.

Reisen, V. A., Abraham, B., \& Toscano, E. M. (2000). Parametric and semiparametric estimations of stationary univariate ARFIMA models. Brazilian Journal of Probability \& Statistics, 14, 185-206.

Riley, M. A., \& VAn ORden, G. C. (EDS.) (2005). Tutorials in contemporary nonlinear methods for the behavioral sciences [Online]. Retrieved October 7, 2008, from www.nsf.gov/sbe/bcs/pac/nmbs/nmbs.jsp.

Shumway, R. H., \& STOFFER, D. S. (2006). Time series analysis and its applications: With $R$ examples. New York: Springer.

Sowell, F. (1992). Maximum likelihood estimation of stationary univariate fractionally integrated time series models. Journal of Econometrics, 53, 165-188.

Stadnytska, T., \& Werner, J. (2006). Sample size and accuracy of estimation of the fractional differencing parameter. Methodology, 4, $135-144$.

Stroe-Kunold, E., \& Werner, J. (2008). Modeling human dynamics by means of cointegration methodology. Methodology, 4, 113-131.

TAqqu, M. S., \& TeVerovsky, V. (1998). On estimating the intensity of long-range dependence in finite and infinite variance time series. In R. J. Adler, R. E. Feldman, \& M. S. Taqqu (Eds.), A practical guide to heavy tails: Statistical techniques and applications (pp. 177-217). Boston: Birkhäuser.

TaquU, M. S., Teverovsky, V., \& Willinger, W. (1995). Estimators for long-range dependence: An empirical study. Fractals, 3, 785-798.

Thornton, T. L., \& Gilden, D. L. (2005). Provenance of correlations in psychological data. Psychonomic Bulletin \& Review, 12, 409-441.

Torre, K., Delignières, D., \& Lemoine, L. (2007a). Detection of long-range dependence and estimation of fractal exponents through ARFIMA modelling. British Journal of Mathematical \& Statistical Psychology, 60, 85-106.

Torre, K., DeligniÈres, D., \& Lemoine, L. (2007b). 1/f fluctuations in bimanual coordination: An additional challenge for modeling. $E x$ perimental Brain Research, 183, 225-234.

Van Orden, G. C., Holden, J. G., \& Turvey, M. T. (2003). Selforganization of cognitive performance. Journal of Experimental Psychology: General, 132, 331-350.

Van Orden, G. C., Kello, C. T., \& Holden, J. G. (in press). Situated behavior and the place of measurement in psychological theory. Ecological Psychology.

WAGENMAKers, E.-J., FARRell, S., \& Ratcliff, R. (2004). Estimation and interpretation of $1 / f^{\alpha}$ noise in human cognition. Psychonomic Bulletin \& Review, 11, 579-615.

WagenMakers, E.-J., FARRELl, S., \& RatClifF, R. (2005). Human cognition and a pile of sand: A discussion on serial correlations and selforganized criticality. Journal of Experimental Psychology: General, 134, 108-116.

WARD, L. M., \& RichaRd, C. M. (2001). 1/f $\alpha$ noise and decision complexity. Unpublished manuscript, University of British Columbia, Vancouver, British Columbia, Canada.

West, B. J., \& Shlesinger, M. F. (1990). The noise in natural phenomena. American Scientist, 78, 40-45.

Zbilut, J. P., \& Marwan, N. (2008). The Wiener-Khinchin theorem and recurrence quantification. Physics Letters A, 372, 6622-6626.

\section{NOTES}

1. Because of this and because the output of Disp, as it is implemented in R, cannot be immediately interpreted by the applied researcher but requires further transformation (see Delignières et al., 2006, for improvements of this approach), this method will not be evaluated here.

2. Delignières et al. (2006) found unsatisfactory estimates of $H$ in $\mathrm{fBm}$ series by means of DFA. Even though fBm series are not under investigation here, bridge detrending might improve how this method handles those series. 


\section{APPENDIX}

\# $* * * * * * * * * * * * * * * * * * * * * * * * * * * * * * * * * * * * * * * * * * * * * * * * * * *$
\# $\mathrm{R}$ Code for the Analysis of the Empirical Examples \#
$\#$ (temporal estimation task and glacial varve series) \#
$\#$ in Stroe-Kunold, Stadnytska, Werner, and Braun:
$\#$ Estimation of long-range dependence in time series: \#
$\#$ an evaluation of estimators implemented in R
$\# * * * * * * * * * * * * * * * * * * * * * * * * * * * * * * * * * * * * * * * * * * * * * * * * * * *$

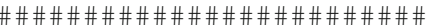

\# package: xlsReadWrite \#

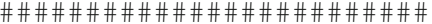

y=read.xls ( "D: / temp_estimation.xls")

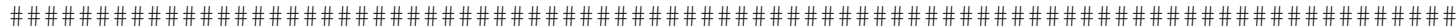
\# as PSD is not implemented in R, the additional code PSD. R has to be loaded \# \# available at: http://www.psychologie.uni-heidelberg.de/ae/meth/stroe-kunold.html \#

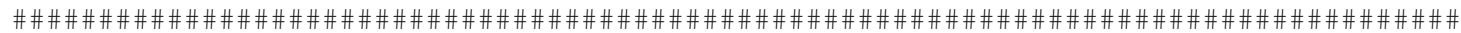
\# PSD analysis

$\operatorname{PSD}(y \$ x)$

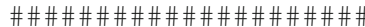

\# package: fracdiff \#

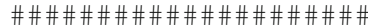

\# fdML estimator for $\operatorname{ARFIMA}(0, \mathrm{~d}, 0)$

fracdiff (y\$x)

\# faML estimator for $\operatorname{ARFIMA}(1, \mathrm{~d}, 1)$

fracdiff (y\$x, nar=1, nma=1)

\# fagPH estimator

fdGPH ( $\mathrm{y} \$ \mathrm{x})$

\# fasperio estimator

fdsperio ( $\mathrm{y} \$ \mathrm{x})$

\# fdWhittle estimator

FDWhittle (y $\$ x)$

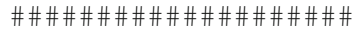

\# package: fractal \#

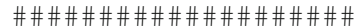

\# Rovers estimator

Rovers (y $\$ x)$

\# hurstipec estimator

hurstspec (y $\$ x)$

\# Higuchi estimator

hurstBlock (y $\$ x$, method="higuchi")

\# DFA estimator

DFA (y\$x, detrend="bridge", sum. order=1)

(Manuscript received June 10, 2008;

revision accepted for publication March 4, 2009.) 